Results: Among the 117 patients with RP, 5 (4.3\%) and 6 (5.1\%) patients had GD and HT, respectively. Patients with RP were more likely to be complicated with GD $\left(p=1.04 \times 10^{-3}\right.$, OR: $\left.7.15,95 \% \mathrm{Cl} 2.68 \sim 18.14\right)$ but not with HT $(p=0.50$, $95 \% \mathrm{Cl} 0.59 \sim 1.27$ ), compared with prevalence in general Japanese population $\left(0.62 \%\right.$ and $5.9 \%$, respectively $\left.{ }^{2}\right)$. RP patients with GD showed a trend to have nasal involvement ( $100 \%$ vs $45.5 \%, \mathrm{p}=0.023$, OR: $2.58,95 \% \mathrm{Cl} 1.09 \sim \infty)$. We did not observe any differences in clinical manifestation in patients with RP and HT. HLA- DPB $1{ }^{*} 02: 02$ demonstrated a trend toward GD complication ( $20 \%$ vs $2.3 \%$, $\mathrm{p}=0.035$, OR: $10.41,95 \% \mathrm{Cl} 1.23 \sim 65.38)$. There were no association of HLA in the complication of HT among patients with RP.

Conclusion: Patients with RP have high co-occurrence ratio of GD. Patients with the two diseases may be characterized by nasal involvement and HLA-DPB $1{ }^{*} 02: 02$

References:

[1] Kung AW et al. Graves' ophthalmopathy and relapsing polychondritis. Clin Exp Rheumatol. 1995 Jul-Aug;13(4):501-3.

[2] Nagataki $S$ et al. Thyroid diseases among atomic bomb survivors in Nagasaki. JAMA. 1994 Aug 3;272(5):364-70.

Disclosure of Interests: Toshiki Nakajima Speakers bureau: Bristol-Myers Squibb and Novartis, Hajime Yoshifuji Grant/research support from: Astellas Pharma. (Outside the field of the present study.), Speakers bureau: Chugai Pharmaceutical. (Outside the field of the present study.), Yoshihisa Yamano: None declared, Hiroshi Handa: None declared, Koichiro Ohmura Grant/ research support from: Astellas Pharma, AYUMI Pharmaceutical, Chugai Pharmaceutical, Daiichi Sankyo, Eisai, Japan Blood Products Organization, Mitsubishi Tanabe Pharma, Nippon Kayaku, Nippon Shinyaku, Sanofi, and Takeda Pharmaceutical., Speakers bureau: AbbVie, Actelion Pharmaceuticals Japan, Asahi Kasei Pharma, AYUMI Pharmaceutical, Bristol-Myers Squibb, Chugai Pharmaceutical, Eisai, Eli Lilly and Company, GlaxoSmithKline, Janssen Pharmaceutical, Mitsubishi Tanabe Pharma, Novartis Pharma, and Sanofi., Tsuneyo Mimori: None declared, Chikashi Terao Grant/research support from: Actelion, Speakers bureau: Asteras, Asahi Kasei Pharma, Ono and Tanabe-Mitsubishi

DOI: 10.1136/annrheumdis-2020-eular.5349

\section{THU0024 METHYLATION ANALYSIS OF VITAMIN D SIGNALING PATHWAY GENES IN RHEUMATOID ARTHRITIS PATIENTS}

E. Punceviciene ${ }^{1,2,3}$, J. Gaizevska ${ }^{4,5}$, R. Sabaliauskaite ${ }^{5}$, L. Venceviciene ${ }^{6,7}$, D. Vitkus ${ }^{8,9}$, S. Jarmalaite ${ }^{4,5}$, I. Butrimiene ${ }^{1,2,3}$. ${ }^{1}$ Centre of Rheumatology, Vilnius University Hospital Santaros Klinikos, Vilnius, Lithuania; ${ }^{2}$ State Research Institute Centre for Innovative Medicine, Vilnius, Lithuania; ${ }^{3}$ Clinic of Rheumatology, Traumatology Orthopaedics and Reconstructive Surgery, Institute of Clinical Medicine of the Faculty of Medicine, Vilnius University, Vilnius, Lithuania; ${ }^{4}$ Institute of Biosciences, Life Sciences Center, Vilnius University, Vilnius, Lithuania; ${ }^{5}$ Laboratory of Genetic Diagnostics, National Cancer Institute, Vilnius, Lithuania; ${ }^{6}$ Clinic of Internal Medicine, Family Medicine and Oncology, Faculty of Medicine, Vilnius University, Vilnius, Lithuania; ${ }^{7}$ Centre of Family Medicine, Vilnius University Hospital Santaros Klinikos, Vilnius, Lithuania; ${ }^{8}$ Institute of Biomedical Sciences of the Faculty of Medicine, Vilnius University, Vilnius, Lithuania; ${ }^{9}$ Centre of Laboratory Medicine, Vilnius University Hospital Santaros Klinikos, Vilnius, Lithuania

Background: Vitamin D is known for its immunomodulatory and epigenome interacting effects. Vitamin $D$ deficiency is frequently observed in rheumatoid arthritis (RA) patients compared to healthy controls, is also named as a potential risk factor in RA ethiopatogenesis and may alter DNA methylation of certain genes [1,2]. Still, causality of vitamin $D$ deficiency in RA patients needs to be elucidated.

Objectives: The aim of the study was to evaluate relationship between DNA methylation status of vitamin D related genes (VDR, CYP24A1, CYP2R1), miRNA-155 expression, vitamin D level and its association with RA.

Methods: $\mathrm{CpG}$ islands in promoter region of the VDR, CYP24A1, CYP2R1 genes were chosen for DNA methylation analysis by means of pyrosequencing. DNA from blood mononuclear cells of 31 RA patients and 31 age and sex matched healthy controls was assessed for methylation pattern after informed consent was obtained in Vilnius university Hospital Santaros klinikos Centre of Rheumatology. For miRNA analysis quantitative reverse transcription PCR was used. Chemiluminescent microplate immunoassay was used to asses $25(\mathrm{OH})$ D serum levels.

Results: $25(\mathrm{OH}) \mathrm{D}$ concentrations varied from deficiency $(<50 \mathrm{nmol} / \mathrm{l})$, insufficiency $(50-75 \mathrm{nmol} / \mathrm{l})$ to normal range $(\geq 75-100 \mathrm{nmol} / \mathrm{l})$ in RA (mean $47.49 \mathrm{nmo}$ $\mathrm{l} / \mathrm{l} ; \mathrm{SD} \pm 27.93$ ) and healthy controls (mean $57.38 \mathrm{nmol} / \mathrm{l} ; \mathrm{SD} \pm 29.93)$ ). CYP24A1 methylation level was significantly higher in comparison to VDR $(\mathrm{p}<0.0001)$ and CYP2R1 $(p<0.0001)$ genes in both groups. CYP24A1 hypermethylation was also observed in older subjects $(p=0.012)$. The study demonstrated a significant positive correlation between vitamin $\mathrm{D}$ concentration and VDR, CYP2R1 genes methylation intensity $\left(r^{2}=0.31, p=0.014 ; r^{2}=0.25, p=0.042\right.$, respectively) However, gene methylation frequency and methylation intensity showed no significant difference between RA patients and healthy controls (VDR - 2.4 vs 2.6 \%, CYP24A1 - 16.6 vs $15.3 \%$, CYP2R1 - 2.6 vs $2.6 \%$ ( $>>0.05)$. To note, miRNA-155 expression negatively correlated with CYP24A1 methylation inten sity $\left(r^{2}=-0.43, p=0.009\right)$.

Conclusion: Our study identified significant associations between the VDR and CYP2R1 promoter methylation and vitamin D concentration. However no significant differences in DNA methylation pattern between RA patients and healthy controls were detected. MiR-155 expression was associated with CYP24A1 methylation level, confirming its possible involvement in vitamin D metabolism. The data of our study suggests that epigenetic phenomena are significantly involved in vitamin D metabolism and may have an indirect effect on RA ethiopatogenesis.

References:

[1] Jeffery LE, et al. Nat Rev Rheumatol. 2016,12.4:201.

[2] Fetahu IS et al. Front Physiol. 2014,5:164

Acknowledgments: This project has received funding from the Research Council of Lithuania (LMTLT), agreement No. S-MIP-17-12.

Disclosure of Interests: None declared

DOI: 10.1136/annrheumdis-2020-eular.2593

\section{THU0025 MICRO-RNA DIFFERENTIALLY REGULATE THE ALTERNATIVE PRTN3-MRNA IN GRANULOMATOSIS WITH POLYANGIITIS}

N. Reichard ${ }^{1}$, A. Kerstein-Staehle ${ }^{1}$, A. Müller ${ }^{1}$, G. Riemekasten ${ }^{1}$, P. Lamprecht ${ }^{1}$, S. Schinke $1 .{ }^{1}$ University Lübeck, Rheumatology and Clinical Immunology, Lübeck, Germany

Background: Micro-RNAs (miRNA) are short non-coding RNAs that regulate inflammation mostly by translational repression. Previously, we screened 847 miRNAs in nasal tissue from GPA patients and found a disease associated alteration of miRNA expression compared to healthy controls and chronic rhinosinusitis. MiR-184 was most over expressed in nasal tissue from GPA (13.4x). The dual-luciferase reporter assay confirmed a significant reduction of Proteinase-3 (PRTN3) expression by miR-184 (1).

PRTN3 transcripts with an alternative 3' untranslated region (UTR) have been described in GPA (2). The pathophysiological relevance of this alternative transcript remains unclarified.

Objectives: To identify new miRNA targets of potential pathophysiological relevance in GPA, we validated the effect of the 21 most dysregulated miRNAs on the mRNA of PRTN3. Further, we included the alternative PRTN3 mRNA in our screen to look for new regulatory differences.

Methods: The inhibitory capacity of miRNAs on Proteinase-3 mRNA was estimated by a dual-luciferase reporter system. The sequences of the alternative (132bp longer) and the regular 3'UTR-PRTN3 were cloned and inserted into the pmirGLO vector and co-transfected with 21 miRNA mimics into HeLa cells. Co-transfection with Caenorhabditis elegans miRNA 67 mimic (cel-miR-67) was used as negative control. Statistical significance was evaluated by students t-test adjusted for multiple comparisons (Holm-Sidak).

Results: For 18 of 21 investigated miRNAs no effects could be observed on the alternative and the regular 3'UTR-PRTN3. But there were remarkable differential effects of let-7f, miR-184 and miR-708. Let-7f (-29,2\%) and miR-708 (-23,6\%) both showed a suppression of the alternative 3'UTR-PRTN3 but no effect on the regular 3'UTR-PRTN3 while miR-184 only suppressed the regular 3'UTR (-17,5 $\%)$ and not the alternative variant (fig. 1-2).

Conclusion: Disease specific miRNA signatures together with an increased PRTN3 level and in alternative PRTN3 mRNA in GPA suggest a dysregulation of PRTN3 expression in GPA. To our knowledge this is the first analysis in GPA showing that miRNAs can differentially regulate the expected and the alternative 3'UTR variants of PRTN3-mRNA. As miR-184 is markedly upregulated in GPA, a repression of PRTN3 is to be anticipated, possibly as a reaction to previous neutrophil activation with PRTN3 overexpression. Our findings also strengthen the potential pathophysiological role of the alternative PRTN3 mRNA.

\section{References:}

[1] Schinke $S$ et al PROTEINASE-3 REGULATING MICRO-RNA IN GRANULOMATOSIS WITH POLYANGIITIS. Ann Rheum Dis 2019 (78 Suppl 2):437 
[2] Mclnnes E et al Dysregulation of Autoantigen Genes in ANCA-Associated Vasculitis Involves Alternative Transcripts and New Protein Synthesis J Am Soc Nephrol. 2015 26(2): 390-399

\section{PRTN3}

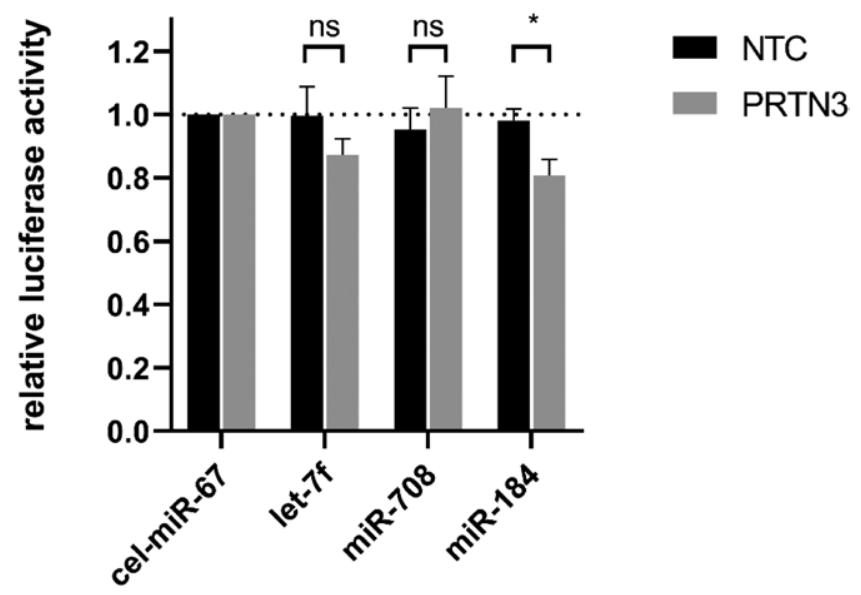

Fig. 1. Dual-luciferase reporter assay with the regular 3'UTR of PRTN3 cloned into the pmirGLO vector compared to empty vector (NTC). Significant effect for miR-184 (17,5\%), miR-708 no effect and let-7f small but not significant reduction in luciferase activity $(12,3 \%)$. Data represent 3 independent experiments with triplicate measurements. miR-184 was tested 6 times. ${ }^{*} \mathrm{P}<0.05 ;$ ns $=$ not significant; error bars display standard deviation.

\section{PRTN3 with alternative 3'UTR}

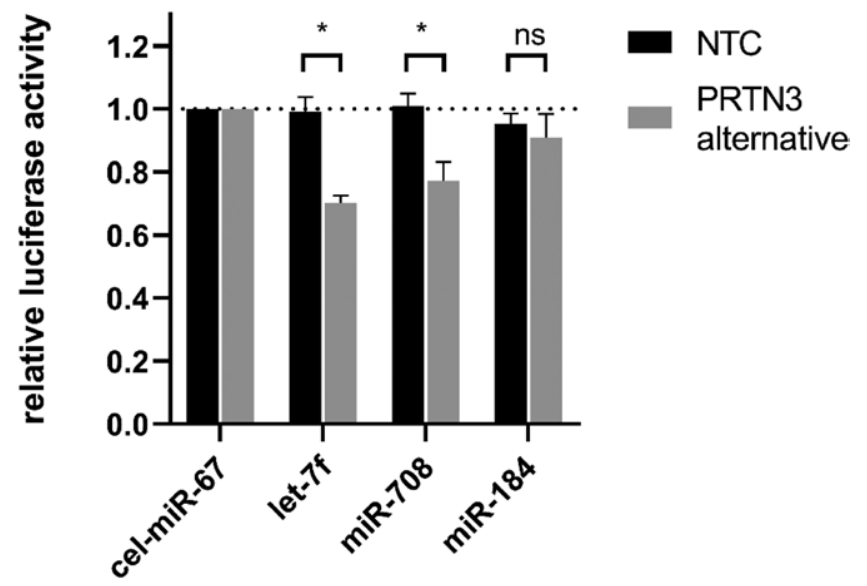

Fig. 2. Dual-luciferase reporter assay with the alternative 3'UTR of PRTN3. Significant effects of let-7f $(29,2 \%)$ and miR-708 mimic $(23,6 \%)$ but no significant effects of miR-184 of luciferase activity. 3 independent experiments with triplicate measurements. ${ }^{*} \mathrm{P}<0.05$

Acknowledgments: Vasculitis foundation for funding

Disclosure of Interests: Nick Reichard: None declared, Anja Kerstein-Staehle: None declared, Antje Müller: None declared, Gabriela Riemekasten Consultant of: Cell Trend $\mathrm{GmbH}$, Janssen, Actelion, Boehringer Ingelheim, Speakers bureau: Actelion, Novartis, Janssen, Roche, GlaxoSmithKline, Boehringer Ingelheim, Pfizer, Peter Lamprecht: None declared, Susanne Schinke Speakers bureau: Pfizer DOI: 10.1136/annrheumdis-2020-eular.4700

\section{THU0026 CLONAL HAEMATOPOIESIS ASSOCIATED SOMATIC MUTATIONS IN RHEUMATOID ARTHRITIS}

F. Tariq ${ }^{1}$, B. Alobaidi ${ }^{2}$, M. Xavier ${ }^{3}$, M. Mccorkindale ${ }^{3}$, J. Veltman ${ }^{4}$, J. Isaacs ${ }^{5}$, A. Pratt ${ }^{6}$, A. Anderson ${ }^{5}$, M. Collin ${ }^{3,6}$ on behalf of Human Dendritic Cell Lab, Newcastle University, United Kingdom. ${ }^{1}$ Newcastle University, Translational and Clinical Research Institute, Newcastle, United Kingdom; ${ }^{2}$ Newcastle University, Newcastle, United Kingdom; ${ }^{3}$ Newcastle University, Newcastle, United Kingdom; ${ }^{4}$ Biosciences, Newcastle, United Kingdom; ${ }^{5}$ Translational and Clinical Research Institute, Newcastle, United Kingdom; ${ }^{6}$ Translational and Clinical Research Institute, Newcastle, United Kingdom
Background: Clonal haematopoiesis of indeterminate potential (CHIP) occurs when somatic mutations arise in myeloid neoplasia driver genes of haematopoietic progenitor cells, in the absence of overt cytopenia or dysplasia. The prevalence of CHIP increases with age. The most common genes affected by CHIP mutations in unselected populations are DNMT3A, ASXL1, and TET2. The presence of $\mathrm{CHIP}$ is linked to increased basal level of inflammation and a high risk of cardiovascular disease and all-cause mortality. Rheumatoid arthritis (RA) is one of the most common and debilitating multi-system autoimmune disorders, affecting up to $1 \%$ of adults in developed countries. The role of somatic mutations in the pathogenesis of autoimmune diseases is an unexplored area; therefore, we aimed to test the hypothesis that clonal haematopoiesis $(\mathrm{CH})$ is associated with the incidence and severity of RA.

Objectives: To evaluate the association of $\mathrm{CH}$ somatic mutation with severity of RA.

Methods: 163 RA patients were recruited from the following cohorts: (i) Early RA/ treatment naive $(n=31)$, (ii) Refractory RA - non-responders to Disease-Modifying Anti-Rheumatic Drugs (DMARDs) and biologics $(n=48)$, (ii) Flare $(n=41)$ vs Remission patients $(n=43)$-patients treated with DMARDs and withdrawn from treatment on achieving remission. Six months later, 50\% relapse and $50 \%$ sustain remission. Single molecule molecular inversion probes (smMIPs) were used to screen for somatic mutations in $\mathbf{4 0}$ loci known to carry clonal haematopoiesis driver mutations (CHDMs). Whole exome sequencing was also performed on Flare/Remission patients $(n=84)$ to screen for CHDMs and other somatic mutations. In-house bioinformatics pipelines were used to call mutations from both the datasets.

Results: We identified $\mathrm{CH}$ in RA with an overall prevalence of $14 \%$. Twenty-four unique variants with a variant allele frequency (VAF) of $2-35 \%$ were found in ten genes including ASXL1, CBL, DNMT3A, GNAS, GNB1, PTPN11, PTPN12, SF3B1, TET2, and TP53. The number of unique patients carrying mutations in these genes are follows: refractory: $n=12 / 48$, flare: $n=6 / 41$, remission: $n=4 / 43$ and early RA: $n=2 / 31$. The majority of the mutations occurred in DNMT3A $(n=6)$ followed by TP53 $(N=4)$ and TET2 $(n=3)$. Two variants with VAF of $15 \%$ were identified in two patients under the age of 30 , both with clinically severe disease. In patients between the ages of 50-59 yrs., 60-69 yrs., and 70-79 yrs., $\mathrm{CH}$ was observed at 11\% (4/35), 23\% (11/46) and $17 \%(7 / 41)$, respectively.

Conclusion: We here report the prevalence of $\mathrm{CH}$ in $\mathrm{RA}$, affecting more patients with clinically advanced/refractory disease compared to those with early/less severe disease.

Further study will be conducted to confirm the results.

References:

[1] Acuna-Hidalgo, R., Sengul, H., Steehouwer, M., van de Vorst, M., VermeuIen, S., \& Kiemeney, L. et al. (2017). Ultra-sensitive Sequencing Identifies High Prevalence of Clonal Hematopoiesis-Associated Mutations throughout Adult Life. The American Journal Of Human Genetics, 101(1), 50-64. doi: 10.1016/j.ajhg.2017.05.013

[2] NRAS - National Rheumatoid Arthritis Society. (2020). Retrieved 30 January 2020, from https://www.nras.org.uk/what-is-ra-article

[3] Steensma, D., Bejar, R., Jaiswal, S., Lindsley, R., Sekeres, M., Hasserjian, R., \& Ebert, B. (2015). Clonal hematopoiesis of indeterminate potential and its distinction from myelodysplastic syndromes. Blood, 126(1), 9-16. doi: 10.1182/blood-2015-03-631747

Acknowledgments: National Institute for Health Research, United Kingdom Disclosure of Interests: Fareeha Tariq: None declared, Bilal Alobaidi: None declared, Miguel Xavier: None declared, Michael McCorkindale: None declared, Joris Veltman: None declared, John Isaacs Consultant of: AbbVie, Bristol-Myers Squibb, Eli Lilly, Gilead, Janssen, Merck, Pfizer, Roche, Arthur Pratt Grant/ research support from: Pfizer, GSK, Amy Anderson: None declared, Matthew Collin: None declared

DOI: 10.1136/annrheumdis-2020-eular.5568

\section{THU0027 THE ASSOCIATION OF THE RS35677470 DNASE1L3 GENE POLYMORPHISM WITH SLE, RA AND SSC:} STRUCTURAL/BIOLOGICAL INSIGHTS

M. Zervou ${ }^{1}$, A. Andreou ${ }^{2}$, C. Matalliotaki ${ }^{3}$, T. Niewold $^{4}$, G. Goulielmos ${ }^{1}$ E. Eliopoulos ${ }^{2}{ }^{1}$ University of Crete, School of Medicine, Heraklion, Greece; ${ }^{2}$ Agricultural University of Athens, Department of Biotechnology, Athens, Greece; ${ }^{3}$ Aristotle University of Thessaloniki, Third Department of Obstetrics and Gynecology, Thessaloniki, Greece; ${ }^{4}$ NYU School of Medicine, Colton Center for Autoimmunity, New York, Greece

Background: Genome-wide association studies (GWAS) have identified hundreds of autoimmune diseases-associated loci so far but much of the heritability of these diseases remains unknown. In an attempt to identify potential causal variants, various studies revealed that the missense variant rs35677470 at DNASE1L3 is associated with the development of systemic lupus erythematosus 